\title{
Effects of Multipath Electrical Stimulation on the Functional Recovery of Early Stage Patients of Total Knee Arthroplasty
}

\author{
Min-Young Lee, PT, MS • Young-Jun Shin, PT, MS • Myoung-Kwon Kim, PT, PhD ${ }^{\dagger}$ \\ Dept. of Physical Therapy, College of Rehabilitation Sciences, Daegu University
}

Received: January 12, 2018 / Revised: January 29, 2018 / Accepted: February 7, 2018

(C) 2018 J Korean Soc Phys Med

\begin{abstract}
| Abstract |
PURPOSE: This research was intended to investigate the influence to function recovery at the early stage after surgery, by conducting Multipath Electrical Simulation and isometric exercise treatment as early stage medical treatment method for Total knee arthroplasty patients.
\end{abstract}

METHODS: The subject of 30 patients having Unilateral Total knee arthroplasty over age 65, Multipath Electrical Simulation and isometric exercise (experiment group I ), Conventional Electrical Simulation and isometric exercise (experiment group II) and isometric exercise (control group). The intervention was performed in 5 times per a week and 60 minutes per a day during 4 weeks. We performed research by conducting Neuromuscular Electrical Stimulation and isometric exercise together and measured pain, range of motion, muscle strength and gait ability before and after intervention.

RESULTS: The result showed therapeutic improvement in

†Corresponding Author : Myoung-Kwon Kim

skybird-98@hanmail.net, http://orcid.org/0000-0002-7251-6108

This is an Open Access article distributed under the terms of the Creative Commons Attribution Non-Commercial License (http://creativecommons.org/licenses/by-nc/3.0) which permits unrestricted non-commercial use, distribution, and reproduction in any medium, provided the original work is properly cited. experiment group I, experiment group II and control group, but Multipath Electrical Simulation and isometric exercise showed significant improvement in function recovery of early stage compared to Conventional Electrical Simulation and isometric exercise, only isometric exercise.

CONCLUSION: Based on research result, in order for early state function recovery of Total knee arthroplasty patients, when conducting neuromuscular electrical stimulation and isometric exercise together, especially when applying Multipath Electrical Stimulation, we could know that it showed more significant improvement to function recovery after surgery. Also, we suggest that Multipath Electrical Simulation may become a useful tool as a method for intervention and performing in various diseases for weakening of Quadriceps muscle.

Key Words: Arthroplasty, Electrical stimulation, Recovery of function

\section{Introduction}

Degenerative arthritis is a disease which shows Degenerative change by damage of Articular cartilage due to aging, overuse, external injury and damage on bone and 
ligament. It is a disease of the highest frequency among diseases which invades joint and especially, it break out mostly at knee joint where the weight is loaded. Degenerative arthritis is the most common degenerative joint musculoskeletal disease that causes physical disability (Koo and Na, 2016). The representative symptom of this disease is abnormal contractile action due to Quadriceps weakness (Rudolph et al., 2007) and it may break out even after the surgery (Fisher et al., 1991; McArdle et al., 2002).

Total knee arthroplasty is known as be very helpful to improve function of daily life of Degenerative arthritis patients such as reducing pain, improving movability and correcting bowed legs(Steven-Lapsley et al., 2012a; Gauchard, 2010). But, Hoogeboom et al. (2015) insisted that complete recovery of muscle function after surgery might be difficult, considering that most patients are in their 60s. It is very important to recover reduced muscular strength into normal state level. Since long time ago, various symptoms were occurred due to joint instability and muscle weakness and efficient plan should be suggested in order to solve weakened muscle function after surgery. Lower extremity strengthening exercise is known to be effective in patients with knee Degenerative arthritis (Park and Kim, 2017). According to research of Meier et al. (2008), muscular strength and activity of Quadriceps muscle immediately after surgery was reduced $5 \sim 20 \%$ compared to before surgery and muscle atrophy occurred. Also, Wylde et al. (2011) said that it causes hypofunction of joint accompanying severe pain due to strong mechanical pressure after incision and surgery. As the muscular strength of Quadriceps muscle is closely related to daily life function movement (Mizner et al., 2005) and if the functional disorder of muscle is not improved, it may lead to reduction daily life activity such as fall down, it is very important to recover reduced muscular strength into normal state level and intensive care should be conducted immediately after surgery (Bade et al., 2010). Researches on various strengthening of muscle and rehabilitation of muscular endurance according to importance of rehabilitation at the beginning stage of surgery aimed at Total knee arthroplasty are consistently performed (Bade and Stevens-Lapsley, 2011). Neuromuscular Electrical Stimulation discharges minute electric currents and causes muscular contraction and it can increase load to muscle through repetitive contraction and increase muscular strength (Huber et al., 2015). After surgery, simultaneous contraction is necessary to improve balance, stability of knee joint medial \& lateral, and as muscular strength balance between vastus medialis and vastus lateralis at knee joint is very important, it is being used. According to preceding research of Multipath Electrical Simulation, Bade and Stevens-Lapsley (2011) conducted research aimed at Total knee arthroplasty applying traditional exercise and Multipath Electrical Simulation, and only the group applying Neuromuscular Electrical Stimulation showed significant improvement in muscle strength, functional ability, knee joint range of motion and simultaneously, it applied Electrical Simulation to Quadriceps muscle only, but we could check the muscular strength of Hamstring muscle was improved. Feil et al. (2011) showed that it has excellent stability of joint and efficiency such as recovering muscular strength compared to Multipath Electrical Simulation, from the research comparing the effect of using Conventional Electrical Simulation and Multipath Electrical Simulation together with traditional exercise aimed at Anterior cruciate ligament surgery patients. Morf et al. (2015) showed more significant result to increasing muscular strength and reduction of muscle fatigue in Multipath Electrical Simulation group, from the result of comparison according to using Conventional Electrical Simulation and Multipath Electrical Simulation aimed at early stage of Total knee arthroplasty patients. According to preceding research, Neuromuscular Electrical Stimulation showed effective result in reducing pain of knee joint and functional improvement such as strengthening muscle, reducing 
muscle fatigue and increasing joint stability of Quadriceps muscle. It applied Neuromuscular Electrical Stimulation in vastus medialis among Quadriceps muscle selectively and suggested efficiency and it proved that Multipath Electrical Simulation is more efficient method with various result such as maximum myotility, pain, balance, function evaluation via comparison of Conventional and Multipath Electrical Simulation. Therefore, in order to investigate the influence of Multipath Electrical Stimulation to recovery of function to early stage patients of Total knee arthroplasty, this research would like to investigate the influence to increasing recovery of function by comparing Multipath Electrical Simulation method applied to Quadriceps muscle with Conventional Electrical Simulation, isometric exercise. In order to reinforce limitation of general rehabilitation method, this research performed two Neuromuscular Electrical Stimulations and conducted knee joint pain, range of motion, muscle strength, gait ability evaluation, so it would like to achieve objective data by quantitatively analyzing the influence and efficiency to improve recovery of function of Total knee arthroplasty patients.

\section{Methods}

\section{Participants}

This research was conducted from January 2017 to April 2017 aimed at 30 people who understood the purpose of this research and agreed to participate in the experiment among the patients who performed Total knee arthroplasty due to Chronic degenerative arthritis and received medical treatment at a general hospital located in Andong City in Gyeongsangbuk-do. This research was performed after getting approval from Institutional Review Board of Daegu University (IRB number is 1040621-201703-HR-008-02), and the criteria to select the subject of research is 1) The person whose age is over 65 regardless of gender, 2) The patient who was diagnosed as degenerative arthritis and received unilateral Total knee arthroplast, 3) The patient who received the 1 st replacement surgery except for redo-replacement, 4) The patient who is in early stage by receiving Total knee arthroplasty within 10 days ago and who does not have complications such as bleeding or infection, 5) The person whose surgery status does not influence to gait or balance, 6) The person who has no neurologic damage which may influence to balance or gait except for Total knee arthroplasty, 7) The person who is fully aware of the purpose and method of experiment and the patient and his/her family's consent was achieved among the patients who didn't skip physical therapy no longer than 3 days due to infection or side effect after surgery, and the criteria for exclusion is 1) The person who has experience of other orthopedic surgery in lower extremity, 2) The person who has cardiovascular disorder, neurologic disorder or other disorder which gives hindrance in activity, 3) The person whose visual status influences to walk or balance, 4) The person who has paralysis in Central nervous system and Peripheral nerve, 5) The person who puts in pacemaker.

\section{Intervention}

The intervention method of this research is divided into 3 groups, the control group is Quadriceps muscle active isometric exercise group and this group is applying 15 minutes in Interference Current Therapy (ICT) as conservative physical therapy, 30 minutes in Continuous Passive Motion (CPM) and 15 minutes in Quadriceps muscle active isometric exercise. By setting up therapy time same as experimental group, Continuous Passive Motion (CPM) is set up as 30 minutes with one cycle from supine to flexion and extension motion starting from $0^{\circ}$ to possible angle in repeated motion and apply up to $120^{\circ}$ maximum according to the status of patient's knee. Interference Current Therapy (ICT) is attached by utilizing 4-pole arrangement method in Quadriceps muscle medial $\&$ lateral and the application time is 15 minutes. Quadriceps 
isometric exercise maintains the angle between maximum extension of knee joint and ankle joint as $90^{\circ}$ in sitting position. At this time, perform the contraction in maximum times for 10 seconds and repeat it 10 times. After rest of 50 seconds, perform the next set and perform it in 15 minutes. Multipath Electrical Simulation group uses Kneehab XP 412 (Bio-Medial Research Ltd, Bio-Medial Research Ltd, China) for Multipath Electrical Simulation, performs CPM and ICT under same condition with control group and then applies Quadriceps muscle active exercise together with Multipath Electrical Simulation. For application of Multipath Electrical Simulation, from sitting position, bend hip joint $85 \sim 90^{\circ}$ knee joint $40 \sim 60^{\circ}$ and then put the feet on the support comfortably from the floor, attach pole set (4 units: $10 \times 20 \mathrm{~cm}, 3 \times 18 \mathrm{~cm}, 10 \times 7.5 \mathrm{~cm}$, $7 \times 14 \mathrm{~cm})$ on kneehab band and cover kneehab band on femur above patella. 4 pads are attached suitably in anatomical location of Quadriceps muscle and adjust the strength inside and outside via electric switch. The strength is applied of the maximum contraction strength which the subject patient can endure within the range of 0-200 mA and it is conducted during 15 minutes (Morf et al., 2015; Walls et al., 2010). At the time of electric stimulus, this experiment was performed after giving sufficient explanation to the subject patient to apply maximum force in knee joint and maintain the extension stance and then maintain the extension status as it is when the stimulus time is over.

Conventional Electrical Simulation group uses Microstim-K13034 (MEDEL GmbH, Germany) for Conventional Electrical Simulation, CPM and ICT under same condition with control group and then applies Quadriceps muscle active exercise together with Conventional Electrical Simulation. For application of Conventional Electrical Simulation, from sitting position, bend hip joint $85 \sim 90^{\circ}$ knee joint $40 \sim 60^{\circ}$ and then attach one pole on small intestine of thigh front part rectus femoris (nerve motor point) and attach other pole at vastus medialis motor point. Electric stimulus is applied with the rate of contraction : non-contraction to be $5: 10$ and applied for 15 minutes, the strength will be the maximum contraction strength that the subject of experiment can endure (Morf et al., 2015). In case of electric stimulus, the experiment was performed after giving sufficient explanation to the subject of experiment to maintain the extension by applying maximum force at knee joint and to maintain the extension status as it is when the stimulus is finished.

\section{1) Outcome measures}

(1) General characteristic of subject of research

30 people participated in this research. These people were designated as 3 group with 10 people, the experiment I performed Multipath Electrical Simulation and isometric exercise, experiment group II performed single path nerve root electric stimulation and isometric exercise and control group performed only isometric exercise.

For randomization, sealed envelopes were prepared in advance and marked inside with $\mathrm{A}, \mathrm{B}$ or $\mathrm{C}$ representing experiment I, II and CG, respectively. This randomization was performed by a third party unaware of the nature of the study. Study characteristics and all outcome measures obtained before and after treatment were assessed by Physician 1, who was blinded to treatment allocations. Intervention was performed in a closed room by Physician 2, who was not involved in subject assessment.

General characteristic of subject of research showed no statistically significant difference between each groups ( $p$ $>.05$ ).

(2) Comparison of Visual Analogue Scale

In order to measure subjective pain of thigh muscle Quadriceps muscle of the leg received knee joint replacement surgery, Visual Analogue Scale (VAS) was used. Make the status of no pain which the subject of experiment can feel at the surgery area as 0 and make the unbearable status of extreme pain as 10 , and on a line 
of $10 \mathrm{~cm}, 0$ is marked on the left side and maximum pain 10 is marked on the right side and the subject can indicate on the line directly and then measure the space and digitize the value (Brodie et al., 1990). The assessed reliability was proved as $95 \%$ in high scale of reliability and sensitivity (Bijur et al., 2003). For control group and experiment group, quantitative evaluation of pain was measured by minimizing fatigue feeling after finishing exercise and taking rest for 30 minutes before and after experiment during research period of 4 weeks.

\section{(3) Comparison of joint range of motion}

In order to measure the range of motion of knee joint of the leg received knee joint replacement surgery, we used range of motion measuring instrument (BPMpro Technology, Brown Spring, USA). This is a tool to visualize the movement of joint and it is printed out on PC screen in $3 \mathrm{D}$ animation. For measurement of knee joint range of motion, we measure 2 movements of bending/extension and the data is printed out via sensor attached on the lower part of leg, and the range of motion is measured in which the heel's moving toward hip. BPMpro sensor indicates "Patient Incidence Points (PIPs)" at the specific location where the patient feels pain or inconvenience and we can check it via monitor.

\section{(4) Comparison of muscular strength}

In order to measure muscular strength of Quadriceps muscle, femoris posterior of the leg which received knee joint replacement surgery, we used digital manual muscle dynamometer (Commander Muscle Tester, JTECH Medical Industries, USA). This is a measuring tool with high reliability (ICC=.96) and validity to measure muscular strength of knee joint (Arnold et al., 2010) and it is very useful for clinical test because it is small-sized and easy to use. For the posture of experiment and using manual muscle dynamometer, we performed the method that Bolgla et al. (2011) conducted before. In order to measure maximal voluntary isometric contraction (MVIC) force of thigh Quadriceps muscle, we made the patients to sit on a chair and bend articulation coxae in $90^{\circ}$ and bend knee joint in $60^{\circ}$, and then we put a small-sized dynamometer in the middle of both ankle bone and lower part of shinbone, not giving pressure to thigh Quadriceps muscle, and fixed it with strap not to be easily moved. In order to measure maximal voluntary isometric contraction force of hamstring, we measured it from prone position. We recorded the measuring location and tried to maintain consistency for every experiment and taught manually the position before the experiment and measured 3 times for interval of contraction for 5 second and rest for 50 seconds same as the research of Walls et al. (2010). The measuring unit is Newton metre (N.m) (Berry et al., 2004; Lee DR et al., 2010).

\section{(5) Change of gait}

For gait test, we measured the characteristic of gait by using gait analyzer (GAITRite, CIR system Inc, USA) in order to measure gait factor of subjects of research. Via GAITRite system, we measured time-based gait variable and spatial gait variable among the characteristic of gait. Gait analyzer is an electronic walking panel with length of $460 \mathrm{~cm}$ and width of $89 \mathrm{~cm}$ and it collects the data for time and spatial variance due to 12 sensors attached in every $30 \mathrm{~cm}$ on the walkway. Collected data was handled by GAITRite GOLD, Version 3.2b software(CIR system Inc, USA, 2007). For this research, the subject experiment maintained standing posture in front of walking panel by using walker and we measure the subject's walking to the opposite side under convenient walking speed according to oral signal of researcher, the measurement is repeated for 3 times and we chose the average value for research data. The inter-measurer reliability of this experiment is $\mathrm{r}=.90$ and intra-measurer reliability is $\mathrm{r}=.96$ (McDonough et al., 2001). 


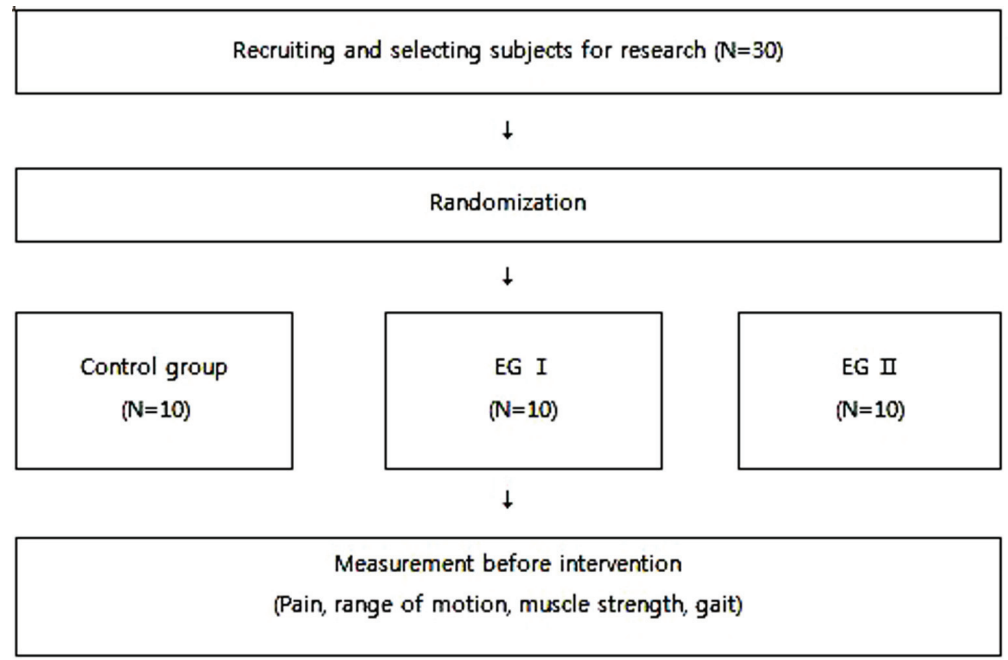

$\downarrow$

Performing intervention (Total 4 weeks, $\mathrm{N}=30$ )

$\downarrow$

Measurement after intervention

(Pain, range of motion, muscle strength, girth of thigh, muscle tone, hardness, elasticity, gait)

$\downarrow$

Analysis of result

(SPSS for windows Ver 18.0)

EG I: Multipath Electrical Stimulation + Isometric exercise

EG II: Singlepath Electrical Stimulation + Isometric exercise

CG: Isometric exercise

Fig. 1. Study flowchart.

\section{2) Statistical Analysis}

We will use SPSS version 18.0 for Windows for statistical handling of the result of this research and the achieved result was described as average \pm standard deviation.

We tested general characteristic between groups of subjects with one-way ANOVA. The difference of pre/post experiment of each group was tested by using Paired t-test and for normality test, Levene's equality of variance was used. In order to compare the difference between groups for variation pre experiment and pre/post experiment, we tested it with one-way ANOVA. And in order to explain the difference between groups post experiment, we performed Bonferroni method for post analysis and conducted statistics process and statistical significance level(a) was set up as .05.

\section{Results}

A flow chart of the study is shown in Fig. 1. The subjects of this research are 30 people. They were designated as 3 groups in 10 people, the experiment I performed Multipath Electrical Simulation and isometric exercise, 
Table 1. General characteristics of the subjects $(n=30)$

\begin{tabular}{ccccccc}
\hline Variable & EG I $(n=10)$ & EG I $(n=10)$ & CG $(n=10)$ & Total $(n=30)$ & $F$ & $p$ \\
\hline Sex (male/female) & $2 / 8$ & $3 / 7$ & $1 / 9$ & $6 / 24$ & & \\
Age (year) & $73.2 \pm 5.9^{\mathrm{a}}$ & $72.2 \pm 8.6$ & $67.4 \pm 9.0$ & $70.9 \pm 8.1$ & .52 & .24 \\
Height $(\mathrm{cm})$ & $155.6 \pm 10.2$ & $157.8 \pm 8.7$ & $157.3 \pm 6.3$ & $156.9 \pm 8.3$ & .18 & .83 \\
Weight $(\mathrm{kg})$ & $58.9 \pm 8.2$ & $60.7 \pm 11.4$ & $61.0 \pm 12.1$ & $60.3 \pm 10.4$ & .11 & .89 \\
\hline
\end{tabular}

$* p<.05$

${ }^{\mathrm{a}} \mathrm{Mean} \pm \mathrm{SD}$

EG I : Multipath Electrical Stimulation+Isometric exercise

EG II: Singlepath Electrical Stimulation+Isometric exercise

CG: Isometric exercise

Table 2. Intra- and inter-group comparisons of outcome measures

\begin{tabular}{ccccccc}
\hline & & EGI $(\mathrm{n}=10)$ & EGII $(\mathrm{n}=10)$ & CG $(\mathrm{n}=10)$ & F & Post-hoc \\
\hline \multirow{2}{*}{ R-VAS } & Pre & $3.70(1.16)$ & $3.40(.70)$ & $3.70(.82)$ & .36 & \\
& Post & $.40(.70)^{*}$ & $1.10(.88)^{*}$ & $1.60(.84)^{*}$ & 5.54 & EGI>CG \\
\multirow{2}{*}{ M-VAS } & Pre & $6.30(1.49)$ & $6.80(.79)$ & $7.00(.82)$ & 1.11 & \\
& Post & $1.50(.71)^{*}$ & $2.60(.84)^{*}$ & $3.70(.95)^{*}$ & 17.19 & EGI>EGII EGI>CG \\
\hline
\end{tabular}

$* p<.05$

Mean \pm SD

R-VAS: VAS in case of resting, M-VAS: VAS in case of moving

EG I : Multipath Electrical Stimulation+Isometric exercise

EG II : Singlepath Electrical Stimulation+Isometric exerciseCG: Isometric exercise

experiment group $I I$ performed single path nerve root electric stimulation and isometric exercise and control group performed only isometric exercise. General characteristic of subject of research is as following Table 1. There was no statistically significant difference between each groups $(p>.05)($ Table 1$)$.

For Visual analogue scale, from the result of paired t-test in each groups for resting and moving cases, the value of all 3 groups was statistically significantly reduced $(\mathrm{p}<.05)$. For comparison between groups, from the result of Visual analogue scale in case of resting, there was statistically significant difference in experiment group I and control group, and the result of Visual analogue scale in case of moving, there was statistically significant difference between experiment group I and experiment group II and experiment group I and control group $(\mathrm{p}<.05)$.

For measuring muscular strength in case of knee joint bending and extension, from the result of paired t-test of each groups, it was statistically significantly increased in all 3 groups $(\mathrm{p}<.05)$. From the inter-groups comparison for measuring knee joint flexor muscle, the result was statistically significantly increased between experiment group I and experiment group II, experiment group II and control group $(\mathrm{p}<.05)$.

From the result of paired t-test of gait velocity within groups, the result was statistically significantly increased in all 3 groups $(\mathrm{p}>.05)$. From inter-groups comparison, there was no statistically significant difference. From the result of paired t-test of single support within groups, the result 
114 | J Korean Soc Phys Med Vol. 13, No. 1

Table 3. Comparison of the ROM among the three condition $(n=30)$

\begin{tabular}{ccccccc}
\hline & & EGI $(\mathrm{n}=10)$ & EGII $(\mathrm{n}=10)$ & CG $(\mathrm{n}=10)$ & F & Post-hoc \\
\hline \multirow{2}{*}{ F-ROM } & Pre & $78.20(19.15)$ & $79.10(17.73)$ & $76.30(19.57)$ & .06 & \\
& Post & $126.50(8.18)^{*}$ & $125.50(7.62)^{*}$ & $122.00(4.83)^{*}$ & 1.13 & \\
& Pre & $2.90(1.10)$ & $3.50(1.08)$ & $2.80(2.15)$ & .61 & \\
\hline \multirow{2}{*}{ E-ROM } & Post & $.20(.42)^{*}$ & $.80(.92)^{*}$ & $1.10(1.10)$ & 2.82 & \\
& & & &
\end{tabular}

$p<.05$

Mean \pm SD

F-ROM: Knee flexion, E-ROM: Knee exetension

EG I : Multipath Electrical Stimulation + Isometric exercise

EG II: Singlepath Electrical Stimulation + Isometric exercise

CG: Isometric exercise

Table 4. Comparison of the STR among the three condition $(n=30)$

\begin{tabular}{ccccccc}
\hline & & EGI $(\mathrm{n}=10)$ & EGII $(\mathrm{n}=10)$ & CG $(\mathrm{n}=10)$ & F & Post-hoc \\
\hline \multirow{2}{*}{ F-STR } & Pre & $9.93(11.77)$ & $26.07(10.89)$ & $11.93(9.32)$ & 2.09 & \\
& Post & $38.45(14.43)^{*}$ & $40.95(8.35)^{*}$ & $27.46(12.32)^{*}$ & 5.49 & EGI>EGII EGII>CG \\
\multirow{2}{*}{ E-STR } & Pre & $15.04(9.58)$ & $28.50(10.72)$ & $19.35(13.75)$ & 1.79 & \\
& Post & $66.88(11.93)^{*}$ & $60.18(14.59)^{*}$ & $44.78(14.16)^{*}$ & 7.17 & EGI> CG \\
\hline
\end{tabular}

$* p<.05$

Mean \pm SD

F-STR: Hamstring muscle, E-STR: Quadriceps muscle

EG I : Multipath Electrical Stimulation+Isometric exercise

EG II: Singlepath Electrical Stimulation+Isometric exercise

$\mathrm{CG}$ : Isometric exercise

was statistically significantly increased in all 3 groups ( $p>.05)$. From inter-groups comparison, there was statistically significant difference in experiment group I and experiment group $I$, experiment group I and control group $(\mathrm{p}>.05)($ Table 2$)$.

\section{Discussion}

This research is a treatment method, set up with active isometric exercise by utilizing Neuromuscular Electrical Stimulation aimed at the patients of early stage knee joint replacement surgery, and it was performed for 4 weeks, in order to investigate the influence on recovery of function after surgery, it was performed by classifying 3 groups, Multipath Electrical Simulation group, single path nerve root electric stimulation group and isometric exercise group, and compared the effect of each group's treatment. After knee joint replacement surgery, as early treatment method, Neuromuscular Electrical Stimulation was performed together with isometric exercise and we could know that there is a difference in speed of recovery of function according to rehabilitation method. From the result of applying Multipath Electrical Simulation in pain, range of motion, muscle strength and gait ability, we could find the most significant influence. 
Effects of Multipath Electrical Stimulation on the Functional Recovery of Early Stage Patients of Total Knee Arthroplasty | 115

Table 5. Comparison of the Gait variable among the three condition $(n=30)$

\begin{tabular}{ccccccc}
\hline & & EGI $(\mathrm{n}=10)$ & EGII $(\mathrm{n}=10)$ & CG $(\mathrm{n}=10)$ & F & Post-hoc \\
\hline \multirow{2}{*}{ Gait velocity } & Pre & $21.56(11.32)$ & $29.10(13.90)$ & $28.90(13.17)$ & 1.12 & \\
& Post & $56.35(13.07)^{*}$ & $57.79(14.11)^{*}$ & $52.05(17.48)^{*}$ & .40 & \\
Single support & Pre & $22.30(6.80)$ & $20.18(5.44)$ & $24.52(10.30)$ & .78 & \\
& Post & $39.99(5.17)^{*}$ & $33.68(4.08)^{*}$ & $33.27(5.85)^{*}$ & 5.48 & EGI>EGII EGI>CG \\
Double support & Pre & $42.47(11.51)$ & $44.76(9.23)$ & $37.46(12.65)$ & 1.11 & \\
& Post & $26.70(4.43)^{*}$ & $28.72(4.65)^{*}$ & $28.82(5.04)^{*}$ & .64 & \\
Cadence & Pre & $41.48(9.39)$ & $54.23(16.84)$ & $56.49(21.62)$ & 2.34 & \\
& Post & $80.50(16.83)^{*}$ & $89.63(20.69)^{*}$ & $83.69(20.23)^{*}$ & .57 & \\
Step length & Pre & $24.53(9.56)$ & $24.29(5.34)$ & $20.56(6.57)$ & .91 & \\
& Post & $47.18(6.70)^{*}$ & $38.33(7.13)^{*}$ & $30.38(7.46)^{*}$ & 14.00 & EGI>EGII EGI>CG \\
\hline
\end{tabular}

$* p<.05$

Mean \pm SD

EG I : Multipath Electrical Stimulation+Isometric exercise

EG II: Singlepath Electrical Stimulation+Isometric exercise

CG: Isometric exercise

Mizner et al. (2005) suggested weakening of muscle activity and muscular strength of Quadriceps muscle as one of the cause of pain among various symptom showing after Total knee arthroplasty and they said that this pain can be reduced through recovery of muscular strength of Quadriceps muscle. From the research by Morf et al. (2015), the result showed that Multipath Electrical Simulation had 39\% lower pain than Conventional Electrical Simulation after knee joint replacement surgery from the measurement result of Visual analogue scale, and from the research by Avramidis and others, it showed significant reduction of pain when applying Neuromuscular Electrical Stimulation to Quadriceps muscle. Therefore, in this research, we desired to check reduction of pain through Neuromuscular Electrical Stimulation and isometric exercise and in order to compare the difference of pain felt by patients according to intervention method and change rate of pain due to intervention method, we measured it by classifying the pain at resting and moving. Before and after medical treatment, pain from all groups was significantly reduced and from the result of comparison of difference between each group, all pain was significantly reduced when resting and moving. During intervention period, no increase of pain due to intervention method showed. Consequently, we can consider that Multipath Electrical Simulation intervention method is clinically effective to adjust pain. From the result of checking variation of range of motion 4 weeks after Total knee arthroplasty by Mizner et al. (2005), there was significant change in knee joint range of motion. This research showed a result supporting preceding research by showing significant increase after 4 weeks.

After surgery, the difference of prognostic can be increased according to status and change of knee joint surrounding muscle. Coombs and Garbutt (2002) said that the imbalance between Quadriceps muscle and Hamstring muscle may cause instability of knee joint and become potential factor which may cause pain of knee joint. According to the preceding research to concentrate on control and balance of Quadriceps muscle and Hamstring muscle, we desired to compare change rate of Quadriceps muscle and Hamstring muscle through muscle which is activated according to nerve root electric stimulation. 
Hence, in this research, we applied Neuromuscular Electrical Stimulation together with isometric exercise and the result showed that the muscular strength was significantly increased from before to after medical treatment in all groups. Application of Neuromuscular Electrical Stimulation medical treatment is applied to knee joint stretching muscle, so stretching muscle showed more improvement than flexor but in experiment group I, we could find more improvement of flexor a lot. In case of attachment of electric stimulation treatment pad is Conventional Electrical Simulation, error on the attaching area can occur. On the contrary, in case of Multipath Electrical Simulation, as the tool is manufactured suitably to anatomical location of Quadriceps muscle, it is possible to attach in accurate location, conduct strong stimulation and possible to adjust strength by classifying inside and outside with dual channel stimulation together with contraction of muscle area, so we could check more effective stimulation delivery and increase of efficiency to realization of joint stability. But, in case of large-sized wound at knee joint replacement surgery, there was a limitation to attach pad. Consequently, in case of Multipath Electrical Simulation in this research, we attached electric pad on knee joint stretching muscle but we could see the effect influencing on increasing flexor muscular strength. Therefore, we could confirm the effect of increasing muscular strength in bending and extension movement of knee joint.

It is said that Total knee arthroplasty patients show reduction of number of strides due to slow gait velocity, reduction of curve angle of knee joint at swing phase and stance phase and walking continuing simultaneous contraction of rectus femoris, Hamstring muscle, gastrocnemius and tibialis anterior for a long time (Benedetti et al., 2003). In this research, in order to investigate improvement of gait ability through Neuromuscular Electrical Stimulation, based on preceding researches, it was researched in order to realize the importance of Quadriceps muscle nerve root electric stimulation and suggest early stage management method on this more efficiently. By measuring time walking variable and spatial walking variable among walking characteristic, from the result of walking analysis, the most significant improvement of walking ability was shown from the experiment group which showed the largest activation of muscle according to complex movement consisted of several muscle movement not a single muscle.

This research applied Neuromuscular Electrical Stimulation treatment in early stage rehabilitation of Unilateral Total knee arthroplasty within 10 days after surgery and made result. For the subjects of this research during medical treatment program period of 4 weeks, we prohibited personal exercise while hospitalization period but we could not actively prohibit all activities of subjects. Also, as the will for rehabilitation is different by each patients, there was difference in activeness of intervention exercise. But, in order to prevent error in experiment and measurement, we tried to minimize error between measures by measuring by 1 person for all measurement before and after intervention. From now on, researches on early stage rehabilitation of Total knee arthroplasty patients, more objective index and functional inspection method is necessary and they should improve health and quality of life of knee joint replacement surgery patients. Even though the importance on strengthening of Quadriceps muscle is being emphasized, there are many researches suggesting to select and reinforce only muscle vastus medialis, a part of Quadriceps muscle and which is known as the weakest muscle and starting muscle weakness early. This is the current situation to need a customized rehabilitation by considering knee muscle strength and arrangement direction of patient, and adjust balance for stabilization of knee joint, not just unconditional training for strengthening muscle vastus medialis.

The limitation of this research is as follows. The number of subjects of this research is few, so we cannot generalize the result of this research into all Quadriceps muscle 
weakened patients. And, we expect that many results, which can be generalized through researches on various knee joint diseases such as knee cartilage, ligament, fracture not only Total knee arthroplasty patients, to be provided as clinical basis to therapist. Also, we expect that various researches and attempts shall be revitalized to apply individual intervention on rehabilitation program for Total knee arthroplasty patients to clinic.

\section{Conclusion}

Based on research result, in order for early stage function recovery of Total knee arthroplasty patients, when conducting Neuromuscular Electrical Stimulation and isometric exercise together, especially when applying Multipath Electrical Simulation, we could know that it showed more significant improvement to function recovery after surgery. Also, we suggest that Multipath Electrical Simulation may become a useful tool as a method for intervention and performing in various diseases for weakening of Quadriceps muscle.

\section{Acknowledgements}

We express our sincere gratitude to those that participated in this study.

\section{References}

Arnold CM, Warkentin KD, Chilibeck PD, et al. The reliability and validity of handheld dynamometry for the measurement of lower-extremity muscle strength in older adults. The Journal of Strength \& Conditioning Research. 2010;24(3):815-24.

Avramidis K, Karachalios T, Popotonasios K, et al. Does electric stimulation of the vastus medialis muscle influence rehabilitation after total knee replacement? Orthopedics. 2011;34(3):175.

Bade MJ, Kohrt WM, Stevens-Lapsley JE. Outcomes before and after total knee arthroplasty compared to healthy adults. Journal of Orthopaedic \& Sports Physical Therapy. 2010;40(9):559-67.

Bade MJ, Stevens-Lapsley JE. Early high-intensity rehabilitation following total knee arthroplasty improves outcomes. Journal of Orthopaedic \& Sports Physical Therapy. 2011;41(12):932-41.

Benedetti M, Catani F, Bilotta T, et al. Muscle activation pattern and gait biomechanics after total knee replacement. Clinical Biomechanics. 2003;18(9): 871-6.

Berry ET, Giuliani CA, Damiano DL. Intrasession and intersession reliability of handheld dynamometry in children with cerebral palsy. Pediatric Physical Therapy. 2004;16(4):191-8.

Bijur PE, Latimer CT, Gallagher EJ. Validation of a verbally administered numerical rating scale of acute pain for use in the emergency department. Academic Emergency Medicine. 2003;10(4):390-2.

Brodie DJ, Burnett JV, Walker JM, et al. Evaluation of low back pain by patient questionnaires and therapist assessment 1. Journal of Orthopaedic \& Sports Physical Therapy. 1990;11(11):519-29.

Bolgla LA, Malone TR, Umberger BR, et al. Comparison of hip and knee strength and neuromuscular activity in subjects with and without patellofemoral pain syndrome. The International Journal of Sports Physical Therapy. 2011;6(4):285.

Coombs R, Garbutt G. Developments in the use of the hamstring/quadriceps ratio for the assessment of muscle balance. Journal of Sports Science Medicine. 2002;1(3):56-62.

Feil S, Newell J, Minogue C, et al. The effectiveness of supplementing a standard rehabilitation program with 
superimposed neuromuscular electrical stimulation after anterior cruciate ligament reconstruction a prospective, randomized, single-blind study. The American Journal of Sports Medicine. 2011;39(6): 1238-47.

Fisher NM, Pendergast DR, Gresham GE, et al. Muscle rehabilitation: its effect on muscular and functional performance of patients with knee osteoarthritis. Archives of Physical Medicine and Rehabilitation. 1991;72(6):367-74.

Gauchard GC, Vançon G, Meyer P, et al. On the role of knee joint in balance control and postural strategies: effects of total knee replacement in elderly subjects with knee osteoarthritis. Gait \& Posture. 2010;32(2): 155-60.

Hoogeboom TJ, van Meeteren NL, Schank K, et al. Risk factors for delayed inpatient functional recovery after total knee arthroplasty. BioMed Research International. 2015.

Huber EO, Roos EM, Meichtry A, et al. Effect of preoperative neuromuscular training (NEMEX-TJR) on functional outcome after total knee replacement: an assessorblinded randomized controlled trial. BMC Musculoskeletal Fisorders. 2015;16(1):1.

Koo HM, Na SS. The short term effects of difference frequency of transcutaneous electrical nerve stimulation on pain relief using c-fos expression in spinal cord with knee osteoarthritis rats. J Korean Soc Phys Med. 2016; 11(4):49-54.

Lee DR, You SH, Lee NG, et al. Strength training-induced changes in muscle size and motor improvement in bilateral schizencephaly: an experimenter-blind case report with 3-month follow-up. Physical Therapy Korea, 2010;17(4):77-87.

Lewek MD, Rudolph KS, Snyder-Mackler L. Quadriceps femoris muscle weakness and activation failure in patients with symptomatic knee osteoarthritis. Journal of Orthopaedic Research. 2004;22(1):110-5.
McArdle A, Vasilaki A, Jackson M. Exercise and skeletal muscle ageing: cellular and molecular mechanisms. Ageing Research Reviews. 2002;1(1):79-93.

McDonough AL, Batavia M, Chen FC, et al. The validity and reliability of the GAITRite system's measurements: A preliminary evaluation. Archives of Physical Medicine and Rehabilitation. 2001;82(3):419-25.

Meier W, Mizner R, Marcus R, et al. Total knee arthroplasty: muscle impairments, functional limitations, and recommended rehabilitation approaches. Journal of Orthopaedic \& Sports Physical Therapy. 2008; 38(5):246-56.

Mizner RL, Petterson SC, Snyder-Mackler L. Quadriceps strength and the time course of functional recovery after total knee arthroplasty. Journal of Orthopaedic \& Sports Physical Therapy. 2005;35(7):424-36.

Morf C, Wellauer V, Casartelli NC, et al. Acute effects of multipath electrical stimulation in patients with total knee arthroplasty. Archives of Physical Medicine and Rehabilitation. 2015;96(3):498-504.

Park JE, Kim SY. Effects of lower extremity strengthening exercise combined with balance exercise on lower extremity function, range of motion, muscle strength, and balance in patients with knee osteoarthritis. J Korean Soc Phys Med. 2017;12(4):147-158.

Rudolph KS, Schmitt LC, Lewek MD. Age-related changes in strength, joint laxity, and walking patterns: are they related to knee osteoarthritis? Physical Therapy. 2007;87(11):1422-32.

Stevens-Lapsley JE, Balter JE, Wolfe P, et al. Early neuromuscular electrical stimulation to improve quadriceps muscle strength after total knee arthroplasty: a randomized controlled trial. Physical Therapy. 2012a;92(2):210-26.

Stevens-Lapsley JE, Balter JE, Wolfe P, et al. Relationship between intensity of quadriceps muscle neuromuscular electrical stimulation and strength recovery after total knee arthroplasty. Physical Therapy. 2012b;92(9): 
$1187-96$.

Walls RJ, McHugh G, O'Gorman DJ, et al. Effects of preoperative neuromuscular electrical stimulation on quadriceps strength and functional recovery in total knee arthroplasty. A pilot study. BMC Musculoskeletal Disorders. 2010;11(1):1.
Wylde V, Rooker J, Halliday L, et al. Acute postoperative pain at rest after hip and knee arthroplasty: severity, sensory qualities and impact on sleep. Orthopaedics \& Traumatology: Surgery \& Research. 2011;97(2): $139-44$. 
